\title{
Huddles and their effectiveness at the frontlines of clinical care: a scoping review
}

\author{
Camilla B. Pimentel, PhD ${ }^{1,2}{ }^{\infty}$, A. Lynn Snow, PhD ${ }^{3,4}$, Sarah L. Carnes, MLIS \\ Nishant R. Shah, $M D^{6,7}$, Julia R. Loup 3,4 , Tatiana M. Vallejo-Luces, $P$ sy $D^{8}$, \\ Caroline Madrigal, $P h D^{9}$, and Christine W. Hartmann, $P h D^{1,10}$
}

\begin{abstract}
'Center for Healthcare Organization and Implementation Research and the New England Geriatric Research, Education and Clinical Center, VA Bedford Healthcare System, Bedford, MA, USA; '2Department of Population and Quantitative Health Sciences, University of Massachusetts Medical School, Worcester, MA, USA; ${ }^{3}$ Alabama Research Institute on Aging and the Department of Psychology, University of Alabama, Tuscaloosa, AL, USA; ${ }^{4}$ Tuscaloosa Veterans Affairs Medical Center, Tuscaloosa, AL, USA; ${ }^{5}$ VA Bedford Healthcare System, Bedford, MA, USA; ${ }^{\circ}$ Division of Cardiology, Department of Medicine, Brown University Alpert Medical School, Providence, RI, USA; ${ }^{7}$ Department of Health Services, Policy and Practice, Brown University School of Public Health, Providence, RI, USA; ${ }^{8}$ Department of Clinical and Health Psychology, College of Public Health and Health Professions, University of Florida, Gainesville, FL, USA; ${ }^{9}$ Center of Innovation in Long Term Services and Supports, Providence Veterans Affairs Medical Center, Providence, RI, USA; ${ }^{10}$ Department of Public Health, Zuckerberg College of Health Sciences, University of Massachusetts Lowell, Lowell, MA, USA.
\end{abstract}

BACKGROUND: Brief, stand-up meetings known as huddles may improve clinical care, but knowledge about huddle implementation and effectiveness at the frontlines is fragmented and setting specific. This work provides a comprehensive overview of huddles used in diverse health care settings, examines the empirical support for huddle effectiveness, and identifies knowledge gaps and opportunities for future research.

METHODS: A scoping review was completed by searching the databases PubMed, EBSCOhost, ProQuest, and OvidSP for studies published in English from inception to May 31, 2019. Eligible studies described huddles that (1) took place in a clinical or medical setting providing health care patient services, (2) included frontline staff members, (3) were used to improve care quality, and (4) were studied empirically. Two reviewers independently screened abstracts and full texts; seven reviewers independently abstracted data from full texts.

RESULTS: Of 2,185 identified studies, 158 met inclusion criteria. The majority $(67.7 \%)$ of studies described huddles used to improve team communication, collaboration, and/or coordination. Huddles positively impacted team process outcomes in $67.7 \%$ of studies, including improvements in efficiency, process-based functioning, and communication across clinical roles (64.4\%); situational awareness and staff perceptions of safety and safety climate (44.6\%); and staff satisfaction and engagement (29.7\%). Almost half of studies (44.3\%) reported huddles positively impacting clinical care outcomes such as patients receiving timely and/or evidence-based assessments and care (31.4\%); decreased medical errors and

Prior Presentations This work was presented on Jun. 3, 2019, at the AcademyHealth Annual Research Meeting in Washington, DC, and on Oct. 9, 2019, at the American Nurses Credentialing Center National Magnet Conference Research Symposium in Orlando, FL.

Supplementary Information The online version contains supplementary material available at https://doi.org/10.1007/s11606-021-06632-9.

Received October 22, 2020

Accepted January 17, 2021

Published online February 8, 2021 adverse drug events (24.3\%); and decreased rates of other negative outcomes $(20.0 \%)$.

DISCUSSION: Huddles involving frontline staff are an increasingly prevalent practice across diverse health care settings. Huddles are generally interdisciplinary and aimed at improving team communication, collaboration, and/or coordination. Data from the scoping review point to the effectiveness of huddles at improving work and team process outcomes and indicate the positive impact of huddles can extend beyond processes to include improvements in clinical outcomes.

STUDY REGISTRATION: This scoping review was registered with the Open Science Framework on 18 January 2019 (https://osf.io/bdj2x/).

KEY WORDS: communication; cooperative behavior; group processes; patient care teams; quality improvement.

J Gen Intern Med 36(9):2772-83

DOI: $10.1007 / \mathrm{s} 11606-021-06632-9$

(C) This is a U.S. government work and not under copyright protection in the U.S.; foreign copyright protection may apply 2021

\section{INTRODUCTION}

Brief, focused, stand-up meetings known as huddles have the potential to improve medical care by enabling collaborative and efficient information exchange and fostering a shared view of current clinical conditions. ${ }^{1}$ Huddles have been shown to minimize hierarchical barriers to care delivery, enhance frontline staff satisfaction, and improve clinical outcomes. ${ }^{2}$ In contrast to historically dominant provider-centric medical practice models, huddles operationalize medicine as a cooperative science: all team members (e.g., physicians, nurses, medical assistants, administrative staff, laboratory workers) work together for the patient's good, ${ }^{3}$ promoting stronger teamwork and communication ${ }^{4}$ and situation awareness on the unit floor. ${ }^{1}$ This increased communication with and among members of the team may also lead to better understanding of 
the daily work of frontline staff, potentially a key to sustaining quality improvement. $^{5}$

Ideally, huddles optimize participant engagement, last 10 $15 \mathrm{~min}$, focus only on essential patient and procedural information, ${ }^{1,6}$ and are held on a regular basis; ${ }^{7}$ in practice, however, huddles take many forms. Some may involve only the patient's immediate clinical team, meeting as needed at the patient's bedside. ${ }^{8}$ Others may involve all clinical and nonclinical staff and be scheduled for the start of each workday or other regular interval. ${ }^{9}$ Huddle structure may also vary, depending, in part, on the use of any facilitation strategies, scripts, or communication tools such as CUS ("I am concerned! I am uncomfortable! This is a safety issue!" ${ }^{10}$ or SBAR (Situation-Background-Assessment-Recommendation). ${ }^{11}$ Yet despite understanding some of the myriad variations in huddle structures and processes, knowledge of huddle implementation and effectiveness at the frontlines of health care remains fragmented and is limited to particular settings. In a preliminary search for relevant reviews available through MEDLINE, the Cochrane Database of Systematic Reviews, and JBI Evidence Synthesis, we found three systematic reviews focusing on the use of huddles: one to promote patient safety in the perioperative setting ${ }^{2}$ and two focused on their use in inpatient settings. ${ }^{12,13}$ A more comprehensive understanding of huddle practices and characteristics has the potential to help clinicians and health care administrators across diverse settings understand how this process can help improve patient care.

In contrast to the prior, narrow systematic reviews on huddles in specific settings, this scoping review thus provides a comprehensive overview of the scope and volume of research on the broad category of clinical-setting huddles that involve frontline staff. In keeping with standard indications for conducting a scoping review, ${ }^{14}$ this review has the following purposes: to describe characteristics of such huddles (e.g., structures, processes), identify empirical support for the effectiveness of huddles for improving health care quality, and highlight knowledge gaps and opportunities for more detailed evidence syntheses and empirical research.

\section{METHODS}

We performed this scoping review using the Joanna Briggs Institute's established method for a scoping review ${ }^{15}$ and guidance from the Preferred Reporting Items for Systematic Reviews and Meta-Analyses Extension for Scoping Reviews (PRISMA-ScR). ${ }^{16}$ The final protocol was prospectively registered with the Open Science Framework ${ }^{17}$ on 18 January 2019 (https://osf.io/bdj2x/) and published in the peer-reviewed literature. $^{18}$

\section{Scope of the Review}

We used the PCC (Population, Concept, and Context) framework to define eligibility criteria. ${ }^{16}$ To be eligible for inclusion, articles had to (1) take place in any clinical or medical setting that provides health care patient services, including inpatient, outpatient, or residential settings; (2) include frontline staff members (i.e., employees with patient contact, including health care providers and non-clinical/administrative staff); ${ }^{19}$ (3) describe, investigate, or explore the huddling practice as a targeted intervention to improve processes and outcomes broadly related to quality of care (e.g., staff engagement and satisfaction, perceptions of safety culture, adverse drug events, patient length of stay); and (4) provide empirical data. Eligible study designs included qualitative studies; experimental and quasi-experimental studies (e.g., randomized or non-randomized controlled trials, before and after studies, interrupted time-series studies); analytic observational studies (e.g., prospective or retrospective cohort studies, case-control studies); and descriptive cross-sectional studies. Dissertations, gray literature, and conference proceedings that met inclusion criteria were also considered. We excluded study protocols; articles that described huddles as a platform through which other interventions were disseminated; articles that solely focused on adherence to a checklist (e.g., surgical safety checklist); simulation studies, and research summaries lacking original data.

\section{Data Sources and Searches}

To be as comprehensive as possible, we performed an initial limited search of PubMed and CINAHL Plus with Full Text, followed by an analysis of each identified article's title, abstract, Medical Subject Headings (MeSH) terms, and keywords. For our full search, we used all resulting relevant MeSH terms and keywords to search the following databases: PubMed, EBSCOhost (including CINAHL Plus with Full Text, Dentistry \& Oral Sciences Source, ERIC, Health Business Elite, Health Policy Reference Center, PsycArticles, PsycBooks, Psychology and Behavioral Sciences Collection, PsycINFO, Rehabilitation \& Sports Medicine Source, Social Work Reference Center, and SocINDEX with Full Text), ProQuest (including the Family Health Database, Health \& Medical Collection, Health Management Database, Nursing \& Allied Health Database, Psychology Database, and PTSDpubs), and OvidSP. Appendix 1 lists the full search strategy used for PubMed, CINAHL Plus with Full Text, EBSCOhost, ProQuest, and OvidSP. We augmented the full database search by scanning the reference list of the Institute for Healthcare Improvement's (IHI's) white paper that guides health care practitioners in using daily huddles as part of a quality management system ${ }^{5}$ for additional articles that we then assessed. Studies published in English from inception to May 31, 2019, were considered for inclusion.

\section{Study Selection}

All articles identified during the full database and additional searches were uploaded into EndNote X8.2 (Clarivate Analytics, Philadelphia, PA, USA), and duplicates were removed. 
We conducted an initial screening for inclusion based on titles and abstracts. Two reviewers (C.B.P., C.W.H.) independently conducted the first screening of the abstracts based on the inclusion criteria to identify articles to include for further review. Disagreements on article inclusion were resolved by discussion or with input from two additional reviewers.

Following this, we reviewed the full text of those articles that met initial screening. Two independent reviewers assessed each article against the inclusion criteria, with disagreements resolved through discussion or with input from a third reviewer. Full-text articles that did not meet the inclusion criteria were excluded from the scoping review. Results of the search are presented in a PRISMA flow diagram (Fig. 1). ${ }^{16}$

\section{Data Abstraction and Quality Assessment}

Seven reviewers independently abstracted relevant data from each full-text article meeting all inclusion criteria. An Excel spreadsheet was used to collect data about, for example, clinical setting, study design, huddle purpose, participating staff, and indicators of huddle effectiveness. The data abstraction tool is available in Appendix 2. To summarize huddle purpose, we began with an adapted list of the four benefits of huddles as outlined by the IHI (engage, update, recognize, and identify $)^{20}$ and added two additional ones based on our findings (plan, provide) (Table 1).

Two secondary reviewers (C.B.P., C.W.H.) independently abstracted relevant data from all full-text articles to assess consistency with primary reviewers. As in the study selection process, disagreements between primary and secondary reviewers were resolved through team discussion or in consultation with a third reviewer. We did not perform formal assessments of methodological quality because a scoping review aims to provide an overview of the existing evidence, irrespective of quality. ${ }^{15,16} \mathrm{We}$ did categorize studies by evidence quality (e.g., peer-reviewed, gray literature, presence of a control comparison group), however, to inform future research.

\section{Role of the Funding Source}

This work was supported by the US Department of Veterans Affairs, Veterans Health Administration, Office of Geriatrics and Extended Care, through the VA Community Living Centers' Ongoing National Center for Enhancing Resources and Training. The funder had no role in study design or conduct, data collection, analysis or interpretation, or reporting.

\section{RESULTS}

We identified a total of 2,185 publications through electronic database searches and reference lists (Fig. 1). After removal of duplicate publications across databases $(N=1,330)$ and exclusion after initial title and abstract review $(N=535)$, we performed full-text review of 325 studies. Two researchers (a primary and secondary reviewer) independently reviewed each article, with 90\% agreement between both reviewers prior to resolution through team discussion or in consultation with a third reviewer. One-hundred fifty-eight studies met inclusion criteria; ${ }^{1,6,8,9,11,21-173} 139(88.0 \%)$ were peerreviewed and 19 (12.0\%) were gray literature. Details related to our broad objectives are summarized below. Select details on the included studies are available in Appendix 3.

\section{Year and Location of Studies}

The first study meeting eligibility criteria was published in $2004 ;{ }^{11}$ the majority $(71.5 \% ; N=113)$ were published between 2014 and $2019 .^{8,9,21,22,25,27,30-32,34-37,39,41-44,47-49,51,53,56-58,62,63,65,67-}$ $70,72,74,76,77,79,81,83,85,87,89,90,92-94,96,97,99-102,104-108,110-113,115-120,122-$ $139,141,142,144,146-150,152-159,161-170,173$ Seventy-four percent $(N=117)$ were performed in the USA, $1,6,8,9,11,21,23,25,27,28,30,33-35,37-40,42-44,46-$ $53,55-62,64,65,69,71,72,74,76-78,84-89,91,93,96-102,104,106,107,109,112,114-126,128-$ $132,135-147,149-153,155,156,158-161,164-170,172,17313.9 \%(N=22)$ in the $\mathrm{UK}^{22,26,29,31,36,41,54,63,67,70,75,79,83,92,94,105,110,111,113,133,154,162}$ $8.9 \%(N=14)$ in Canada, $24,32,45,68,73,80,82,90,103,108,127,134,157,171$ and $3.2 \%$ elsewhere (the Netherlands $[N=2],{ }^{81,163}$ Thailand $[N=1],{ }^{95}$ Israel $[N=1],{ }^{66}$ and Australia $[N=1]^{148}$ ).

\section{Clinical Setting}

A majority $(30.4 \% ; \mathrm{N}=48)$ of all studies described implementation of huddle-based interventions throughout entire hospitals or health care $\mathrm{s}$ y $\mathrm{s}-$

$\mathrm{t}$ e $\mathrm{m}$ -

s. $1,6,21,23,25,28,30,37,39,41,42,47,56,57,62,63,71,73,74,83,87,89,91,94,98,104,105,109,114,1-$ 15,117,123,125,128-131,133,135-137,143,150,160,162,165,169,170 Specific unit-level clinical settings included the following: perioperative settings/ operating room $(15.2 \% ; N=24),{ }^{11,24,26,29,38,45,46,52,59,65,66,70,75,76,78-}$ $82,84,86,95,103,113$ intensive care units $(12.7 \%$; $N=20)^{33,44,50,61,77,88,96,108,110,112,121,124,138,140,151,152,159,163,171,172}$ inpatient medical or surgical departments (13.3\%; $N=21), 35,49,51,54,60,67,85,92,99-101,111,116,118,141,147,154,155,157,168,173$ longterm care facilities $(6.3 \% ; N=10), 31,40,97,120,122,127,132,139,148,166$ primary care $(6.3 \% ; N=10),{ }^{43,55,58,64,68,69,93,142,149,164}$ emergency departments $(5.1 \% ; N=8), 8,90,145,146,151,153,158,161$ and labor and delivery (3.8\%; $N=6$ ). ${ }^{53,72,119,126,156,167}$ Two percent or fewer studies were specific to each of the following settings: neurology and stroke $(N=3),{ }^{34,107,144}$ oncology $(N=2),{ }^{48,98}$ dental $(N=2),{ }^{22,36}$ behavioral health $(N=2),{ }^{27,139}$ pathology $(N=1),{ }^{32}$ radiology $(N=1),{ }^{9}$ cystic fibrosis $(N=1),{ }^{102}$ outpatient echocardiography laboratory $(N=1),{ }^{106}$ and brain and spinal cord rehabilitation $(N=1) .{ }^{134}$

\section{Study Design}

Among peer-reviewed studies, 107 used quantitative methods only and 32 used qualitative or mixed methods. Among studies in the gray literature, 18 used quantitative and 1 used qualitative methods.

Only 9 studies (5.7\%), all from the quantitative peer-reviewed literature, used a comparison group design. $38,49,87,90,115,121,138,141,162$ 


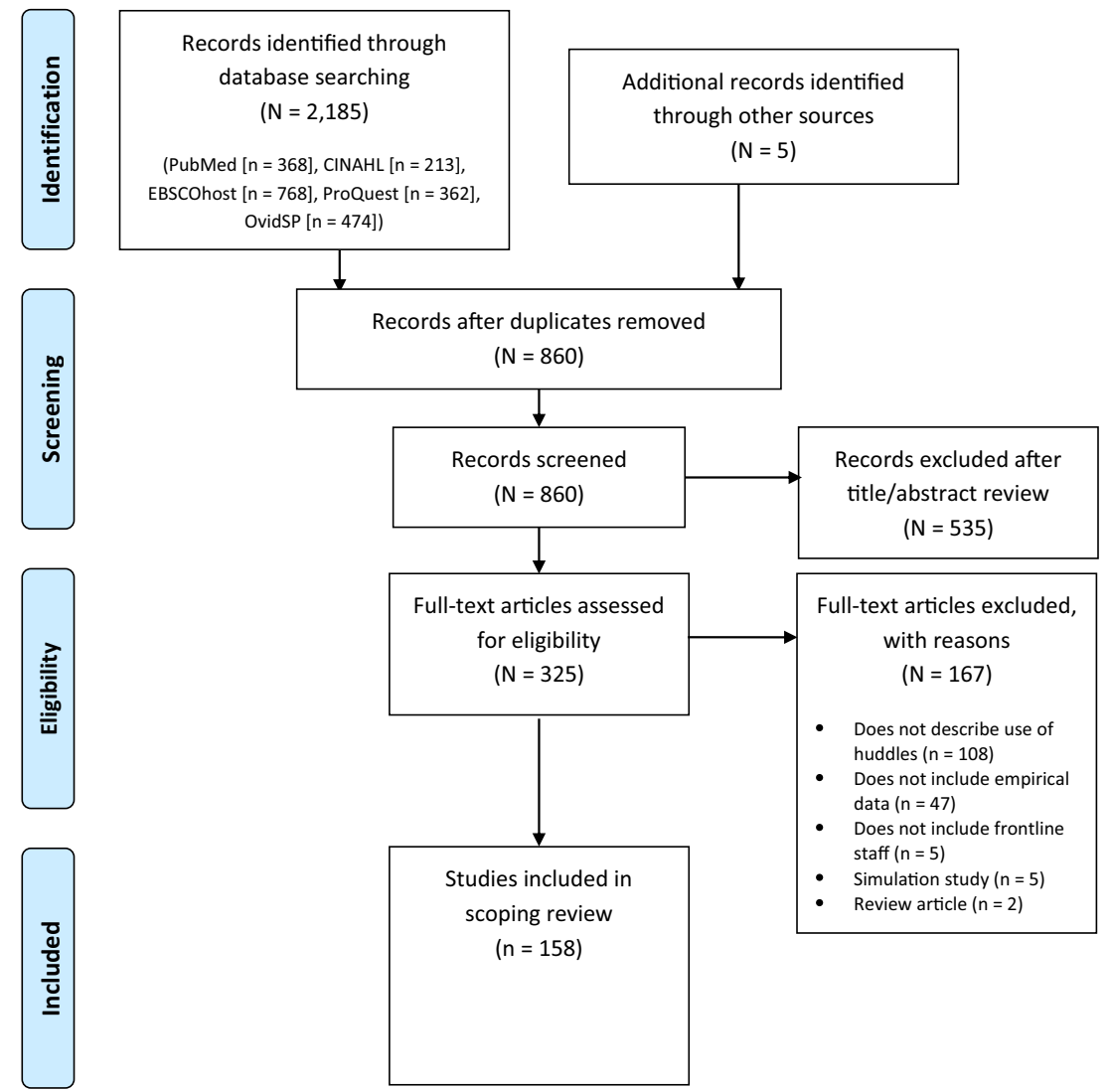

Figure 1. PRISMA flow diagram.

Nearly half of all studies $(N=75)$ were analytic observational $1,23,26,28,31,34,37,41,46-48,50,51,55,58-61,67,71,72,74-$ $80,83,84,86,89,91,94,96,97,99,101,106,107,109-112,115,117,119,123,125,128-130,132$ 134,137,140,142,143,146-148,150,153,157-161,163,166-168,170,172 34.2\% ( $N=54)$ were experimental or quasi-experimental, $8,11,27,29,32,33,35,38,42,43,45,49,51-$ $53,56,65,70,73,81,85,87,90,92,95,102-105,108,113,114,116,118,120-122,124$ $126,131,135,136,138,139,141,144,149,152,155,156,162,165,173$ and $9.5 \%(N=15)$ were descriptive cross-sectional. ${ }^{9,22,30,36,39,40,57,64,66,93,100,127,151,169,171}$ Twenty-one percent of studies $(N=33)$ used qualitative methods, $6,21,24,25,29$ $31,37,43,44,54,55,57,58,62-64,66,68,69,77,80,82,87,88,98,127,142,145,148,154,157,164$ among which half $(N=17)$ used mixed methods. ${ }^{29-}$ $31,37,43,57,58,64,66,68,77,80,87,127,142,148,157$

Huddles were the sole intervention in $42.4 \%$ of studies $(N=67)^{6,8,9,11,24}$ $26,28-30,36,38,40,42-47,51,52,54,55,57-60,62,63,65-68,74-78,80-$ $86,88,89,95,104,105,109,110,113,116,117,123,125,127,130,134,136,138,140,144,153,164,169$ and part of a larger intervention bundle in $57.6 \%(N=91)^{1,21-23,27,31-35,37,39,41,48}$ $50,53,56,61,64,69-73,79,87,90-94,96-103,106-108,111,112,114,115,118-122,124,126,128,129,131-$ $133,135,137,139,141-143,145-152,154-163,165-168,170-173$

\section{Huddle Purpose}

Studies used huddles for multiple purposes (Table 1). The majority $(67.7 \%)$ of studies $(N=107)$ described huddles as being used to engage team members in thinking and talking about their work and to improve communication, collaboration, and/or coordination. ${ }^{6,8,11,21-124}$ Roughly equal numbers of studies described huddles used to identify issues requiring immediate attention or escalation to high-level management for resolution (27.2\%; $N=43)$; , , ,9,21,25,27,29,30,32,37,39,44,53,55,57,59,63,69,75,84,87,93,98,116,122,126,134,144,146,149,153-162,165,167,171 update team members about safety and quality issues that affect their work, including reviewing prior issues $(24.1 \%$; $N=38) ;, 6,29,32,40,57,72,73,77,83,87,89,110,111,113,118,122,123,125-139,167-171,173$ and plan for or improve processes for future work $(22.8 \%$; $N=36)^{9,31,34,50,55,57,59,60,66,69,71,93,100,108,121,122,130,132,134,135,140-}$ 151,166,168,170,172 Fewer studies described huddles used to recognize workrelated issues that may be addressed by training, coaching, or revising tools and methods $(4.4 \% ; N=7)^{40,57,62,89,112,152,169}$ or to provide a framework for running Plan-Do-Study-Act cycles $(1.3 \% ; N=2) .{ }^{163,164}$

\section{Theories and Tools}

More than one-third of the studies $(37.3 \% ; N=59)$ were based on a conceptual rationale, $1,6,11,21,23,27,30-32,35,37,42,47,49,53,54,57,58,61-$ $63,66,67,69,70,73,75,78,83,89,90,94,98,107,115,119-121,123,125-128,132,133,135,145-$ $148,156,159,162-164,166,170,172,173$ such as a theory (why the subject of interest will have an impact) and/or a framework or model (how a theory is operationalized). ${ }^{174}$ Among these studies, the most common were high reliability organizational principles $(17.6 \% ; N=9)$, ${ }^{16,63,69,70,123,125,128,135 ~ c r e w ~}$ resource management $(17.6 \% ; N=9),{ }^{11,54,61,75,78,89,94,98,121}$ and Lean Six Sigma $(15.7 \% ; N=8)$. $27,30,32,49,115,126,163,170$

Only $7.6 \%$ of studies $(N=12)$ mentioned organizing their huddles using existing tools or communication scripts (e.g., SBAR; 
Table 1 Huddle Purpose and Outcome Measures ( $N=158$ Articles)

\section{Huddle purpose \\ Outcomes \\ Related to work and team process} Engage team members in think
and talking about their work; improve communication, collaboration, and/or coordination $^{21-24}$ across clinical roles safety $6,8,11,26,30,31,35-37,40,45-47$,
Improved efficiency, process-based functioning and communication Improved situational awareness and staff perceptions of

Increased staff satisfaction and More supportive practice

climate $^{6,}$

Enhanced self-efficacy among frontline staff to implement evidence-based practices
Other
11,28,29,32,38,42,52,57,61,70,71,75,76,79,86,96-99

Update team members about safety and quality issues that affect their work, including reviewing prior issues $(108,111,119,141,149$, 159)

Plan for future work/improving processes for future work (108, $128,129,149,162$ )

Recognize work-related issues that can be addressed by training, coaching, and revising tools and methods (119)

Identify issues requiring immediate attention or escalation to higherlevel management for resolution $(54,84,111,141)$ across clinical roles

Improved situational awareness and staff perceptions of safety $6,40,57,73,77,83,128,129$

Increased staff satisfaction and engagement $6,77,87,89,127,128,130$

More supportive practice climate

Enhanced self-efficacy among frontline staff to implement Other ${ }^{32,57,125}$

Improved efficiency, process-based functioning and communication across clinical roles

Improved situational awareness and staff perceptions of safety $^{3}$

Increased staff satisfaction and engagement $59,60,69,130,142,148$

More supportive practice climate, 9,50,57,66,69,7

Enhanced self-efficacy among frontline staff to implement

evidence-based practices $34,66,91,93,148$

Other $^{57,71}$

Improved efficiency, process-based functioning and communication across clinical roles

Improved situational awareness and staff perceptions of safety ${ }^{40,57,62}$

Increased staff satisfaction and engagement ${ }^{89}$

More supportive practice climate ${ }^{40,57,62}$

Other $^{57}$

Improved efficiency, process-based functioning and communication across clinical roles Improved situational awareness and staff perceptions of
safety $8,30,37,53,55,57,63,69,75,84,146,153,156$

Increased staff satisfaction and engagement ${ }^{8,30,37,59,63,69,87,156,157}$

More supportive practice climate

Enhanced self-efficacy among frontline staff to implement

evidence-based practices $30,87,93,154$

Other ${ }^{2}$
Improved efficiency, process-based functioning and communication evidence-based practices $29,77,87,127,131$

\section{Related to clinical care}

Increased proportion of patients receiving timely, evidence-based as-
sessment or treatment

Decreased medical errors and adverse events $^{11,25,50,86,109-113}$

Decreased length of hospital stay $^{3}$

Decreased rate of negative outcomes, such as infections, falls, and pressure ulcers 31

Increased patient satisfaction ${ }^{116,117,123}$

Decreased costs ${ }^{116,117}$

Other $^{34,36,51,56,63,72,74,117,124}$

Increased proportion of patients receiving timely, evidence-based assessment or treatment ${ }^{132,133}$

Decreased medical errors and adverse events ${ }^{110,111,113,128,134-136}$

Decreased length of hospital stay ${ }^{118}$

Decreased rate of negative outcomes, such as infections, falls, and pressure ulcers $89,122,125,130,133,137$

Increased patient satisfaction ${ }^{123}$ Other ${ }^{72,138,139}$

Increased proportion of patients receiving timely, evidence-based assessment or treatment ${ }^{100,108,132,149}$

Decreased medical errors and adverse events ${ }^{121,134,135}$

Decreased length of hospital stay ${ }^{144}$

Decreased rate of negative outcomes, such as infections, falls, and pressure ulcers $31,122,130,150$

Increased patient satisfaction ${ }^{141,149}$

Decreased medical errors and adverse events $^{112}$

Decreased rate of negative outcomes, such as infections, falls, and pressure ulcers 89,152

Increased proportion of patients receiving timely, evidence-based assessment or treatment ${ }^{27,30,149,158-160}$ Decreased medical errors and adverse events $1,25,134,161$

Decreased length of hospital

Decreased rate of negative outcomes, such as infections, falls, and pressure ulcers $^{122}$

Increased patient satisfaction 116,117,149 Other ${ }^{1,63,149,155,157,160}$

Decreased medical errors and adverse events $^{163}$

Other ${ }^{164}$

Provide a fram
PDSA cycles

*List of huddle purposes adapted from $\mathrm{IHI}^{20}$

${ }^{\dagger}$ Citations in Huddle Purpose column represent articles that characterized a huddle purpose(s) but did not characterize a specific outcome(s)

Table 2); $8,21,23,65,73,94,101,138,145,146,159,16615.8 \%(N=25)$ developed and published their own huddling tools. ${ }^{27,29,36,42,45,46,49-}$ $51,56,60,68,78,80,87,89,96,98,104,112,130,134,136,141,153$

\section{Participating Staff}

In studies that identified huddle participants' job categories $(N=120)$, nurses were involved in $88.3 \%$ of the studies $(N=106){ }^{1,6,8,9,11,21,24}$ $26,28-30,34-46,49-51,53-60,62,63,65,66,68-73,75,77-81,83-89,91,95,96,98-$ 
Table 2 Common Tools Used to Communicate in or Monitor Frontline Staff Huddles

\begin{tabular}{ll}
\hline \hline Tool & Description \\
\hline CUS ("I am concerned! I am uncomfortable! This & $\begin{array}{l}\text { The CUS assertive statements help frontline staff speak up or speak out in uncomfortable } \\
\text { situations. A script is presented, using "I am concerned," "I am uncomfortable," and "This is a } \\
\text { safety issue." }\end{array}$ \\
$\begin{array}{l}\text { The SBAR technique provides a framework for health care team members to discuss a patient's } \\
\text { condition. "S" is a concise statement of the problem, "B" is pertinent and brief information about } \\
\text { Recommendation) }\end{array}$ & $\begin{array}{l}\text { the situation, "A" is the team member's analysis and considerations of options, and "R" is the } \\
\text { team member's request or recommendation. } \\
\text { The HOT is a participant/non-participant observation tool that may be used to provide objective } \\
\text { measures of (1) huddle effectiveness and (2) changes to huddles, situational awareness, and } \\
\text { collaborative culture over time. }\end{array}$ \\
\hline
\end{tabular}

$150,152,153,155,157-160,162,164,165,173$ physicians in $75.8 \%$ $(N=91)^{0} 6,9,11,24,26,28-30,32,35,36,38-46,49-51,53-59,62,63,65,66,68-73,75,78-81,83-$ 87,91,94-96,98-101,103,104,108-114,117-119,121-123,134,136,140$142,145,146,149,152,153,155,157,158,160,162,164,165,172$ members of ancillary services (e.g., social workers; phamacists; technicians; case managers; respiratory, physical, or occupational therapists) in $50.8 \%(N=61){ }^{11,26,28-30,36}$ $40,45,49,50,54,63,65,66,69-71,75,78,80,81,83-85,89,91,94,96,98-101,103,105,109$ $112,114,117,118,121,122,125,136,142-144,146,149,152,155,157-160,162,164$ managers in $23.3 \%$ $(N=28) ;{ }^{1,6,9,21,25,27,30,32,34,40,44,57,63,69,74,88,89,98,117,123,126,136,143,150,162,164,169,172 ~ a n d ~}$ other frontline staff (e.g., clerical staff, environmental services) in $13.3 \%$ $(N=16))^{930,32,37,55,58,63,68,89,94,98,125,136,162,164,169}$ Many huddles were interdisciplinary in nature, including participants from more than one job category. More than $24 \%$ of all studies $(N=38)$ did not specify participants' job categories. Three percent $(N=5)$ of all studies explicitly included patients and/or their family members and peer supports. 27,30,53,57,125

Nurses, usually charge nurses or nurse managers, facilitated the huddles in $40.0 \%$ of studies $(N=20)^{1,28-}$ $30,43,44,57,60,85,88,89,112,125,129,130,136,138,147,149,150$ where information on facilitator job category was included $(N=52) ., 9,21,22,28-$ $30,33,35,40,43,44,47,51,54,55,57,59,60,68,69,71,83-85,87-89,98$ $100,108,111,112,117,123,125,127,129,130,135,136,138,145,147,149,150,162,164,169-171$

Other huddle facilitators were attending physicians or medical directors $(32.0 \% ; N=16), 9,22,28-30,40,54,55,57,71,84,87,98-100,136$ unspecified administrative leaders (26.9\%; $N=14),{ }^{9,21,30,33,44,47,57,83,88,123,136,150,169,170}$ physician trainees $(13.5 \% ; N=7),{ }^{1,35,51,55,100,108,117}$ unspecified members of the health care team $(13.5 \% ; N=7)^{57,59,68,69,127,145,162}$ or safety team $(7.7 \%$; $N=4),{ }^{47,135,136,171}$ and pharmacists $(6.0 \% ; N=3) .{ }^{111,136,164}$ In $67.1 \%$ $(N=106)$ of all studies, information about huddle leaders was lacking.

Among studies that specified the number of huddle partici $\mathrm{p}$ a $\mathrm{n} \mathrm{t} \mathrm{s}$ $(N=31){ }^{1,8,31,42,45,53,55,58,62,65,68,71,72,82,87,91,100,104,118,127,138,1-}$ $40,142,144,145,148,153,158,163,165,169$ a range of 2 to 20 frontline staff members attended huddles. Nearly $77 \%$ of the studies ( $N=121$ ), however, did not specify the number of participants.

Huddles, when duration was specified, lasted anywhere between 2 and 30 min. Most of these huddles were held either once $(41.1 \%$; $N=53)^{6,9,26,27,29,34,35,39,40,42,45,47,51,55,60,62,67-}$ $69,73,74,77,80,81,85,87,92,93,102,104,108,111,113,114,116-118,123,126,129,131,138,140,142$ $144,149,155,160,162,169,170,172$ or twice $(12.7 \% ; N=20)$ daily, $123,28,33,37,43,44,58,59,88$ $90,120,128,133,145,154,156,157,16024.7 \%(N=39)$ before or after an event of interest (e.g., surgery, fall, activation of sepsis alam), 8,22,24,36,49,53,54,64 66,71,72,78,82$84,91,96,100,105,109,112,119,121,122,130,135-137,141,146,150,152,158,161,165,168,173$ and 8.9\% $(N=14)$ on a weekly basis or more infrequently. ${ }^{30-}$ $32,66,110,127,132,134,147,148,163,164,166,171$ Nearly $18 \%$ of studies $(N=28)$ did not provide this level of detail when describing the huddles.

\section{Effectiveness of Huddles}

All 9 quantitative studies with a control comparison group reported statistically significant improvements associated with huddles. $^{38,49,87,90,115,121,138,141,162}$ Of the 123 quantitative studies without a control comparison group, all but 2 reported improvements. Half ( $N=60)$ of these studies reported positive findings reaching statistical significance. $^{1,27,29,32,33,35,39,41,45,46,51,52,56,58,61,65,71,72,75,78-}$ $81,84,85,91,94,99,101-104,106,109,112,113,116-119,122,125,129,130,134,135,139,142-$ 144,146,148,149,152,155-157,163,165,173 All studies reported at least one outcome, with many reporting multiple process and clinical care outcomes. Of the $63.9 \%(N=101)$ studies measuring work and team process outcomes (Table 1), all but 1 reported that the huddle had a statistically significant positive impact on frontline staff. $6,8,9,25-99,125-$ 131,140-148,150,153-157 Of these, studies found evidence for improved efficiency, process-based functioning, and communication across clinical roles $(64.4 \% ; N=65)$; $^{6,8,9,25-73,125-127,140-145,153-155}$ improved situational awareness and staff perceptions of safety and safety climate $(44.6 \%$; N=45); $6,8,11,26,30,31,35-37,40,45-47,53,55-$ $57,60,62,63,67-69,71,73-86,128,129,145-147,153,156$ increased staff satisfaction and engagement $(29.7 \% ; N=30) ;^{6,8,11,30,33,37,38,41,47,49,58-}$ $60,62,63,68,69,77,79,85,87-89,127,128,130,142,148,156,157$ perceptions of a more supportive practice climate $(26.7 \% ; N=27)$; $6,8,9,11,28,30,40,43,47,49,50,57,58,62,66-69,71,79,84,85,90,91,127,154,156$ and en-

hanced self-efficacy among frontline staff to implement evidencebased practices and/or improve care (20.8\%; $N=21){ }^{29,30,34,38,48,51,54,58,66,71,77,81,87,92-95,127,131,148,154}$ Only 2 qualitative studies (less than $2 \%$ of all studies) specifically assessed and reported on unintended negative consequences of the huddling practice, including added pressure on staff time and workload, ${ }^{22}$ exclusion of clinical trainees, and inadvertent reinforcement of medical hierarchies. ${ }^{24}$ We summarize the major findings on huddle team process outcomes in Fig. 2.

Seventy studies $(44.3 \%)$ measured clinical care outcomes $^{1,11,25,27,30,31,34,36,41,49-51,56,63,72,74,86,89,94,97,99-101,104-}$ $125,128,130,132-139,141,144,149-152,155,157-164$, of which all reported the huddle had a positive clinical impact. Positive 


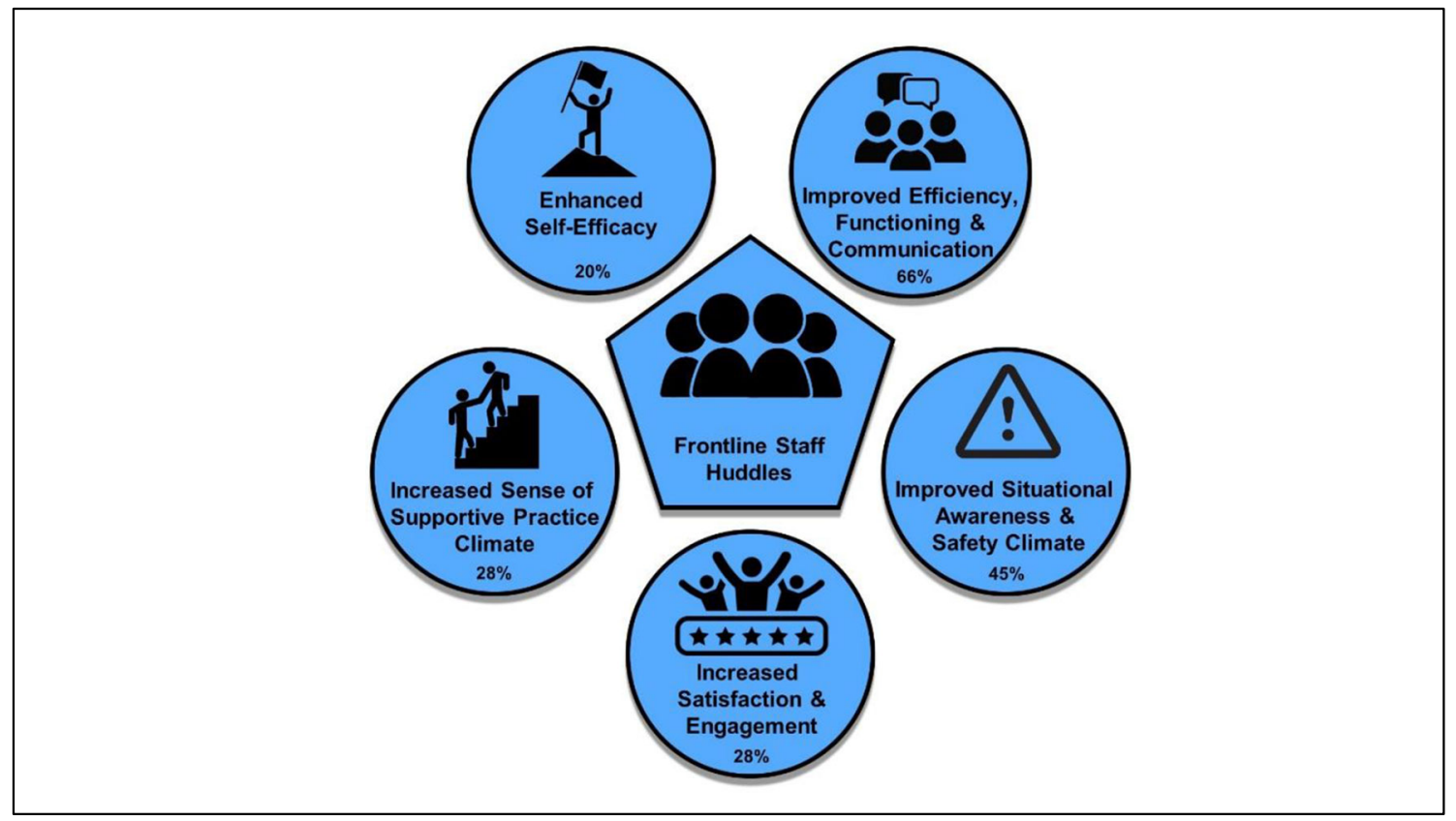

Figure 2 Positive work and team process outcomes associated with frontline huddles (with percent of studies reporting each outcome; $N=101$ ). Studies could report more than one outcome; percent totals are over 100.

clinical care outcomes included the following: increased proportion of patients receiving timely and/or evidence-based assessments or care $(31.4 \% ; N=22) ; 27,30,41,51,94,97,99-$ $108,132,133,149,158-160$ decreased medical errors and adverse drug events $(24.3 \% ; N=17){ }^{1,11,25,50,86,109-113,121,128,134-}$ 136,161,163 decreased rate of other negative outcomes, e.g., infections, falls, and pressure ulcers (20.0\%; $N=14) ; 31,89,94,97,119-122,125,130,133,137,150,152$ decreased length of hospital stay and improvements to discharge-related measures $(15.7 \% ; N=11) ;^{30,49,94,114-118,144,157,162}$ increased patient and family satisfaction $(7.1 \% ; N=5) ;{ }^{116,117,123,141,149}$ and decreased costs $(2.9 \% ; N=2) .{ }^{116,117}$

\section{DISCUSSION}

We undertook this scoping review to provide a comprehensive overview of huddles used in health care, examine the empirical support for huddle effectiveness, and identify knowledge gaps and opportunities for future studies. Findings from our review show that huddles involving frontline staff are an increasingly prevalent practice across diverse health care settings. Huddles are generally interdisciplinary and aimed at improving team communication, collaboration, and/or coordination. Data from our review point to the effectiveness of huddles at improving work and team process outcomes and indicate the positive impact of huddles can extend beyond processes to include improvements in clinical outcomes.

For a huddle to be clearly distinguished as effective, it must (1) be identifiable as a huddle and (2) be linked to a positive outcome. But identification of a huddle may be difficult. This scoping review included articles that self-identified interventions as huddles or that otherwise described quick, "touch base" meetings of healthcare team members, convened by a designated or situational leader, to enhance or regain situational awareness, discuss critical issues and emerging events, anticipate outcomes and likely contingencies, assign resources, and express concerns. ${ }^{2,7}$ But study authors' definitions of huddles varied considerably, owing to heterogeneity of studies' participants and huddle frequency, duration, and purpose. Some practices were labeled as huddles and therefore included in our review but represent conceptually different practices. Handoffs, meaning predominantly one-way transfers of information, ${ }^{7}$ stand in contrast to the majority of studies, where huddle information exchange was more collaborative and engaged. Huddle interventions should also ideally be distinguished from rounds, which are typically didactic, ${ }^{176-178}$ specific to the clinical care and plan for a given patient, and attended primarily by members of that patient's healthcare team (physicians and nurses). Shift change report, when relevant verbal or written information is passed from one shift of workers (e.g., outgoing nurses) to another (e.g., oncoming nurses) to maintain continuity of care and patient safety, ${ }^{179-}$ ${ }^{181}$ should also be a distinct category. In addition, some studies did not link targeted objectives to the study intervention, making it impossible to distinguish whether the huddle was related to positive outcomes. ${ }^{23,166-173}$ These findings point to a need in the literature for improved coherence in studies' definitions of huddles and tighter links between study huddle interventions and outcomes.

Use of published tools may help promote clarity around the huddling process. The Standards for Quality Improvement Reporting Excellence (SQUIRE), ${ }^{182}$ for example, provide guidance on reporting new knowledge about how to improve health care. Another tool is the Huddle Observation Tool, ${ }^{175}$ a 
psychometrically sound observational assessment tool that records team processes occurring during a huddle, enables qualitative measures of huddle effectiveness, and facilitates continuous quality improvement of the huddle itself. Systematic use of these tools will promote standardization of the huddling practice; enable greater understanding of local variations in huddling structure, processes, and implementation strategies; and facilitate intra- and inter-study evaluations of huddle effectiveness.

This review also identified a gap in the literature: the lack of conceptual rationales for implementation of huddle-based interventions. This is consistent with other reviews of quality improvement studies in health care ${ }^{183-185}$ and is despite an extensive menu of relevant theories, models, and frameworks for quality improvement and implementation science. We did not assess whether studies that used an explicit conceptual rationale were of higher quality or were more effective than other studies; however, conceptually grounded interventions are more likely to be widely disseminated. ${ }^{186}$ Ample resources, e.g., Dissemination \& Implementation Models in Health Research \& Practice (https://dissemination-implementation. org/), exist to help health care practitioners and researchers select and apply appropriate conceptual approaches for quality improvement. Use of conceptual approaches provides a better sense of how and why an intervention succeeds or fails; ${ }^{174}$ describes or guides efforts to translate research into practice (i.e., "how-to" process models); and helps evaluate implementation strategies. Greater use of theories, models, and frameworks in future studies on huddles will promote comparison opportunities and, consequently, bring rigor to the growing body of research on the huddling practice.

Many opportunities exist for future research on frontline huddles in healthcare. Researchers should consider experimental study designs comparing a huddle intervention with a control group, as well as quasi-experimental studies assessing the impact of huddles independent of contemporaneous trends or other quality improvement initiatives. This will reduce potential sources of confounding, enhancing the methodological rigor of huddle-based studies. Future research should also specifically evaluate unanticipated consequences associated with the huddling practice, as we only found two studies assessed and reported on negative outcomes. Qualitative research methods, specifically, may enable greater exploration of mechanisms through which huddles may (or may not) impact work and team processes and clinical care outcomes. Finally, implementation studies are needed to facilitate the adoption, scale-up, and maintenance of proven huddle-based interventions in routine healthcare practice.

The potential for reporting (publication) bias represents a limitation of our scoping review. It is possible that practitioners or researchers with positive experiences of or significant findings about huddles submitted more manuscripts for publication and were more successful at being published than those with negative or not significant findings. Heterogeneity of setting, population, study design and methods, and purpose among the large number of studies reviewed also added complexity to our data collection efforts. Nevertheless, it is this same heterogeneity that demonstrates the breadth of clinical settings that have adopted the frontline staff huddling practice and indicates where gaps remain, such as huddle use in nonhospital-based settings (e.g., long-term care) and huddles that incorporate input from non-clinical staff members and patients.

\section{CONCLUSION}

A rich and rapidly growing body of empirical research appears to support the use of huddles in diverse clinical contexts for improving work and team processes and the quality of clinical care. This evidence base would greatly benefit from improvements in the conceptualization and design of studies on huddles and an increase in research on theoretically guided huddle-based interventions.

Acknowledgements: The authors are grateful for Mx. Teddy Bish op's assistance on the bibliography and for Dr. Daniel Okyere and Ms. Sharon Sloup's early contributions to this work.

Corresponding Author: Camilla B. Pimentel, $\mathrm{PhD}$; Center for Healthcare Organization and Implementation Research and the New England Geriatric Research, Education and Clinical Center, VA Bedford Healthcare System, Bedford, MA, USA (e-mail: Camilla. Pimentel@va.gov).

Funding This work was supported by the US Department of Veterans Affairs (VA), Veterans Health Administration, Office of Geriatrics and Extended Care, through the VA Community Living Centers' Ongoing National Center for Enhancing Resources and Training. The funding source had no role in the study's design, conduct, and reporting. The content of this article is solely the responsibility of the authors and does not necessarily represent the official views of the US Department of Veterans Affairs or the United States Government.

\section{Declarations:}

Conflict of Interest: The authors declare no competing interests.

\section{REFERENCES}

1. Brady PW, Muething S, Kotagal U, et al. Improving situation awareness to reduce unrecognized clinical deterioration and serious safety events. Pediatrics. 2013;131(1):e298-308.

2. Glymph DC, Olenick M, Barbera S, et al. Healthcare Utilizing Deliberate Discussion Linking Events (HUDDLE): A Systematic Review. Aana j 2015;83(3): 183-8.

3. Mitchell P, Wynia M, Golden R, et al. Core principles \& values of effective team-based health care. 2012. Accessed at Institute of Medicine at http://www.iom.edu/tbc on Dec 102018.

4. Gittell JH. New directions for relational coordination theory. In: Spreitzer GM, Cameron KS, eds. The Oxford handbook of positive organizational scholarship. Oxford: Oxford University Press; 2012:40011

5. Scoville $\mathbf{R}$, Little $\mathbf{K}$, Rakover J, et al. Sustaining Improvement: IHI White Paper. 2016. Accessed at Institute for Healthcare Improvement at http://www.ihi.org/resources/Pages/IHIWhitePapers/Sustaining-Improvement.aspx on Dec 112018. 
6. Goldenhar LM, Brady PW, Sutcliffe KM, et al. Huddling for high reliability and situation awareness. BMJ Gual Saf 2013;22(11):899906.

7. Agency of Healthcare Research and Quality. Team STEPPS 2.0. 2006. Accessed at Agency of Healthcare Research and Guality at https:// www.ahrq.gov/teamstepps/index.html on Oct 92018.

8. Martin HA, Ciurzynski SM. Situation, Background, Assessment, and Recommendation-Guided Huddles Improve Communication and Teamwork in the Emergency Department. J Emerg Nurs 2015;41(6):484-8.

9. Donnelly LF, Cherian SS, Chua KB, et al. The Daily Readiness Huddle: a process to rapidly identify issues and foster improvement through problem-solving accountability. Pediatr Radiol 2017;47(1):2230 .

10. Agency for Healthcare Research and Quality. CUS Tool - Improving Communication and Teamwork in the Surgical Environment Module. 2017. Accessed at Agency for Healthcare Research and Quality at http://www.ahrq.gov/professionals/quality-patient-safety/hais/tools/ ambulatory-surgery/sections/implementation/training-tools/cus-tool. html on Dec 102018.

11. Leonard M, Graham S, Bonacum D. The human factor: the critical importance of effective teamwork and communication in providing safe care. Qual Saf Health Care 2004; 13 Suppl 1:i85-90.

12. Ryan S, Ward M, Vaughan $\mathbf{D}$, et al. Do safety briefings improve patient safety in the acute hospital setting? A systematic review. J Adv Nurs 2019;75(10):2085-98.

13. Franklin BJ, Gandhi TK, Bates DW, et al. Impact of multidisciplinary team huddles on patient safety: a systematic review and proposed taxonomy. BMJ Qual Saf. Published Online First: 07 April 2020.

14. Munn Z, Peters MDJ, Stern C, Tufanaru C, McArthur A, Aromataris E. Systematic review or scoping review? Guidance for authors when choosing between a systematic or scoping review approach. BMC Med Res Methodol 2018;18:143.

15. The Joanna Briggs Institute. Joanna Briggs Institute Reviewers' manual: 2017 edition. 2017. Accessed at The Joanna Briggs Institute at https://joannabriggs.org/assets/docs/sumari/ReviewersManual2014.pdf on Oct 22018 .

16. Tricco AC, Lillie E, Zarin w, et al. PRISMA Extension for Scoping Reviews (PRISMA-ScR): Checklist and Explanation. Ann Intern Med 2018;169(7):467-73.

17. Open Science Framework. 2011. Accessed at https://osf.io on Jan 18 2019.

18. Pimentel CB, Hartmann CW, Okyere D, et al. Use of huddles among frontline staff in clinical settings: a scoping review protocol. JBI Evid Synth 2020;18(1): 146-53.

19. Kirchner JE, Parker LE, Bonner LM, et al. Roles of managers, frontline staff and local champions, in implementing quality improvement: stakeholders' perspectives. J Eval Clin Pract 2012;18(1):63-9.

20. Institute for Healthcare Improvement. Patient Safety Essentials Toolkit: Huddles. Accessed at http://www.ihi.org/resources/Pages/Tools/Huddles.aspx on May 62020.

21. Baloh J, Zhu X, Ward MM. Implementing team huddles in small rural hospitals: How does the Kotter model of change apply? J Nurs Manag 2018;26(5):571-8.

22. Finn DJ, Rajlawat BP, Holt DJ, et al. The development and implementation of a biopsy safety strategy for oral medicine. Br Dent J 2017;223(9):667-73.

23. Freitag M, Carroll VS. Handoff communication: using failure modes and effects analysis to improve the transition in care process. Qual Manag Health Care 2011;20(2): 103-9.

24. Whyte S, Lingard L, Espin S, et al. Paradoxical effects of interprofessional briefings on OR team performance. Cogn Tech Work 2008; 10(4):287-94.

25. AHC Media LLC. Huddles getting popular, but use them correctly. Healthcare Risk Management; Atlanta. 2014;36(5)

26. Ali M, Osborne A, Bethune R, et al. Preoperative Surgical Briefings Do Not Delay Operating Room Start Times and Are Popular With Surgical Team Members. J Patient Saf 2011;7(3):139-43.

27. Balfour ME, Tanner K, Jurica PJ, et al. Using Lean to Rapidly and Sustainably Transform a Behavioral Health Crisis Program: Impact on Throughput and Safety. Jt Comm J Qual Patient Saf 2017;43(6):27583.

28. Berenholtz SM, Schumacher $\mathbf{K}$, Hayanga AJ, et al. Implementing standardized operating room briefings and debriefings at a large regional medical center. Jt Comm J Qual Patient Saf 2009;35(8):391-7.
29. Bethune R, Sasirekha G, Sahu A, et al. Use of briefings and debriefings as a tool in improving team work, efficiency, and communication in the operating theatre. Postgrad Med J 2011;87(1027):331-4.

30. Bourgault AM, Upvall MJ, Graham A. Using Gemba Boards to Facilitate Evidence-Based Practice in Critical Care. Crit Care Nurse 2018;38(3):e1-e7.

31. Cooper R. Reducing falls in a care home. BMJ Qual Improv Rep. 2017;6(1).

32. Cromwell S, Chiasson DA, Cassidy D, et al. Improving Autopsy Report Turnaround Times by Implementing Lean Management Principles. Pediatr Dev Pathol 2018;21(1):41-7.

33. Dingley C, Daugherty K, Derieg MK, et al. Advances in Patient Safety Improving Patient Safety Through Provider Communication Strategy Enhancements. In: Henriksen K, Battles JB, Keyes MA, Grady ML, eds. Advances in Patient Safety: New Directions and Alternative Approaches (Vol. 3: Performance and Tools). Rockville: Agency for Healthcare Research and Quality (US); 2008.

34. Driscoll M, Tobis $\mathbf{K}$, Gurka D, et al. Breaking down the silos to decrease internal diversions and patient flow delays. Nurs Adm $\mathrm{Q}$ 2015;39(1):E1-8.

35. Khan A, Baird J, Rogers JE, et al. Parent and Provider Experience and Shared Understanding After a Family-Centered Nighttime Communication Intervention. Acad Pediatr 2017;17(4):389-402.

36. Graham C, Reid S, Lord TC, et al. The evolution of patient safety procedures in an oral surgery department. Br Dent J 2019;226(1):32-8.

37. Harris $\mathbf{K}$, Dawson M, Poe T, et al. Nurse communication strategies to improve patient outcomes in a surgical oncology setting. ORL - Head Neck Nurs 2017;35(4):5-12.

38. Henrickson SE, Wadhera RK, Elbardissi AW, et al. Development and pilot evaluation of a preoperative briefing protocol for cardiovascular surgery. J Am Coll Surg 2009;208(6):1115-23.

39. Hughes Driscoll C, El Metwally D. A daily huddle facilitates patient transports from a neonatal intensive care unit. BMJ Qual Improv Rep. 2014;3(1).

40. Hutcheon RG, Iorlano M, Thomas MK. Clinical status: a daily forum for resident discussion and staff education. J Am Med Dir Assoc 2010;11(9):671-6.

41. Iwaniszak C, Rees E, Meal R, et al. Improving care of patients with terminal agitation (TA) at end of life at Leeds Teaching Hospitals NHS Trust. BMJ Support Palliat Care 2018;8:A70.

42. Jain AL, Jones KC, Simon J, et al. The impact of a daily pre-operative surgical huddle on interruptions, delays, and surgeon satisfaction in an orthopedic operating room: a prospective study. Patient Saf Surg 2015;9:8.

43. Kellish AA, Smith-Miller C, Ashton K, et al. Team Huddle Implementation in a General Pediatric Clinic. J Nurses Prof Dev 2015;31(6):324-7.

44. Kylor C, Napier T, Rephann A, et al. Implementation of the Safety Huddle. Crit Care Nurse 2016;36(6):80-2.

45. Lingard L, Regehr G, Orser B, et al. Evaluation of a preoperative checklist and team briefing among surgeons, nurses, and anesthesiologists to reduce failures in communication. Arch Surg 2008;143(1): 127; discussion 8.

46. Makary MA, Mukherjee A, Sexton JB, et al. Operating room briefings and wrong-site surgery. J Am Coll Surg 2007;204(2):236-43.

47. Melton L, Lengerich A, Collins M, et al. Evaluation of Huddles: A Multisite Study. Health Care Manag (Frederick) 2017;36(3):282-7.

48. Mikan SQ, Wilfong LS, Rhoads $\mathbf{M}$, et al. Improvements in communication and engagement of advance care planning in adults with metastatic cancer through a targeted team approach. J Clin Oncol. 2016;34(26_suppl): 14 .

49. Molla M, Warren DS, Stewart SL, et al. A Lean Six Sigma Quality Improvement Project Improves Timeliness of Discharge from the Hospital. Jt Comm J Qual Patient Saf 2018;44(7):401-12.

50. Neily J, Mills PD, Lee $\mathbf{P}$, et al. Medical team training and coaching in the Veterans Health Administration; assessment and impact on the first 32 facilities in the programme. Qual Saf Health Care 2010;19(4):360-4.

51. Newman RE, Bingler MA, Bauer PN, et al. Rates of ICU Transfers After a Scheduled Night-Shift Interprofessional Huddle. Hosp Pediatr 2016;6(4):234-42

52. Nundy S, Mukherjee A, Sexton JB, et al. Impact of preoperative briefings on operating room delays: a preliminary report. Arch Surg 2008; 143(11):1068-72.

53. O'Rourke K, Teel J, Nicholls E, et al. Improving Staff Communication and Transitions of Care Between Obstetric Triage and Labor and Delivery. J Obstet Gynecol Neonatal Nurs 2018;47(2):264-72. 
54. Papaspyros SC, Javangula KC, Adluri RK, et al. Briefing and debriefing in the cardiac operating room. Analysis of impact on theatre team attitude and patient safety. Interact Cardiovasc Thorac Surg 2010;10(1):43-7.

55. Picciano A, Winter RO. Benefits of huddle implementation in the family medicine center. Fam Med 2013;45(7):501-4

56. Prager JD, Ruiz AG, Mooney K, et al. Improving operative flow during pediatric airway evaluation: a quality-improvement initiative. JAMA Otolaryngol Head Neck Surg 2015; 141(3):229-35.

57. Provost SM, Lanham HJ, Leykum LK, et al. Health care huddles: managing complexity to achieve high reliability. Health Care Manag Rev 2015;40(1):2-12.

58. Rodriguez HP, Meredith LS, Hamilton AB, et al. Huddle up!: The adoption and use of structured team communication for VA medical home implementation. Health Care Manag Rev 2015;40(4):286-99

59. Setaro J, Connolly M. Safety huddles in the PACU: when a patient selfmedicates. J Perianesth Nurs 2011;26(2):96-102.

60. Shermont H, Mahoney J, Krepcio D, et al. Meeting of the minds. Nurs Manag 2008;39(8):38-44.

61. Shields R, Taub M, Bender J, et al. Board 257 - Program Innovations Abstract Train to Sustain (Submission \#1282). Simul Healthc 2013;8(6):495.

62. Shunk R, Dulay M, Chou CL, et al. Huddle-coaching: a dynamic intervention for trainees and staff to support team-based care. Acad Med 2014;89(2):244-50.

63. Stapley E, Sharples E, Lachman P, et al. Factors to consider in the introduction of huddles on clinical wards: perceptions of staff on the SAFE programme. Int $J$ Qual Health Care 2018;30(1):44-9.

64. Taliani CA, Bricker PL, Adelman AM, et al. Implementing effective care management in the patient-centered medical home. Am J Manag Care 2013; 19(12):957-64.

65. Tibbs SM, Moss J. Promoting teamwork and surgical optimization: combining TeamSTEPPS with a specialty team protocol. AORN 2014;100(5):477-88

66. Vashdi DR, Bamberger PA, Erez M, et al. Briefing-debriefing: Using a reflexive organizational learning model from the military to enhance the performance of surgical teams. Hum Resour Manag 2007;46(1):115-42.

67. Venkataraman A, Conn R, Cotton RL, et al. 117 Enhancing situational awareness through safety huddles - a staff perspective. Arch Dis Child. 2017;102(Suppl 3):A31-A.

68. Walsh A, Moore A, Everson J, et al. Gathering, strategizing, motivating and celebrating: the team huddle in a teaching general practice. Educ Prim Care 2018;29(2):94-9.

69. Weaver RR. Seeking high reliability in primary care: Leadership, tools and organization. Health Care Manag Rev 2015;40(3):183-92.

70. Williams JP, Spernaes I, Duff E, et al. Development and implementation of a theatre booking form and morning briefing meeting to improve emergency theatre efficiency. J Perioper Pract 2017;27(10):217-23.

71. Wolf FA, Way LW, Stewart $\mathbf{L}$. The efficacy of medical team training: improved team performance and decreased operating room delays: a detailed analysis of 4863 cases. Ann Surg 2010;252(3):477-83; discussion 83-5.

72. Wood J, Stevenson E. Using Hourly Time-Outs and a Standardized Tool to Promote Team Communication, Medical Record Documentation, and Patient Satisfaction During Second-Stage Labor. MCN Am J Matern Child Nurs 2018;43(4): 195-200.

73. Wright JG, Roche A, Khoury AE. Improving on-time surgical starts in an operating room. Can J Surg 2010;53(3):167-70.

74. AHC Media LLC. Safety huddles produce results if they are controlled and monitored. Healthc Risk Manag. 2016;38(10).

75. Allard J, Bleakley A, Hobbs A, et al. Pre-surgery briefings and safety climate in the operating theatre. BMJ Qual Saf 2011;20(8):711-7.

76. Carpenter JE, Bagian JP, Snider RG, et al. Medical Team Training Improves Team Performance: AOA Critical Issues. J Bone Joint Surg Am 2017;99(18):1604-10

77. Chiafery MC, Hopkins P, Norton SA, et al. Nursing Ethics Huddles to Decrease Moral Distress among Nurses in the Intensive Care Unit. J Clin Ethics 2018;29(3):217-26.

78. Gore DC, Powell JM, Baer JG, et al. Crew resource management improved perception of patient safety in the operating room. Am J Med Qual 2010;25(1):60-3.

79. Hill MR, Roberts MJ, Alderson ML, et al. Safety culture and the 5 steps to safer surgery: an intervention study. $\mathrm{Br} \mathrm{J}$ Anaesth 2015;114(6):958-62.
80. Khoshbin A, Lingard L, Wright JG. Evaluation of preoperative and perioperative operating room briefings at the Hospital for Sick Children. Can J Surg 2009;52(4):309-15.

81. Leong $\mathbf{K}$, Hanskamp-Sebregts $\mathbf{M}$, van der Wal RA, et al. Effects of perioperative briefing and debriefing on patient safety: a prospective intervention study. BMJ Open 2017;7(12):e018367.

82. Lingard L, Whyte S, Espin S, et al. Towards safer interprofessional communication: constructing a model of "utility" from preoperative team briefings. J Interprof Care 2006;20(5):471-83.

83. Motuel L, Dodds S, Jones S, et al. Swarm: a quick and efficient response to patient safety incidents. Nurs Times 2017;113(9):36-8.

84. Paige JT, Aaron DL, Yang T, et al. Improved operating room teamwork via SAFETY prep: a rural community hospital's experience. World J Surg 2009;33(6):1181-7.

85. Townsend CS, McNulty M, Grillo-Peck A. Implementing Huddles Improves Care Coordination in an Academic Health Center. Prof Case Manag 2017;22(1):29-35.

86. Wright MA. KP Northwest Preoperative Briefing Project. Perm J 2005;9(2):35-9

87. Bennett NL, Flesch JD, Cronholm P, et al. Bringing Rounds Back to the Patient: A One-Year Evaluation of the Chiefs' Service Model for Inpatient Teaching. Acad Med 2017;92(4):528-36.

88. Hyde P. Implementing the "Cardiac Huddle". Crit Care Nurse 2008;28(2): 144.

89. Lubinensky M, Kratzer R, Bergstol J. Huddle up for patient safety. Am Nurse Today 2015;10.

90. Ginsburg $\mathbf{L}$, Bain $\mathbf{L}$. The evaluation of a multifaceted intervention to promote "speaking up" and strengthen interprofessional teamwork climate perceptions. J Interprof Care 2017;31(2):207-17.

91. Paige JT, Aaron DL, Yang T, et al. Implementation of a preoperative briefing protocol improves accuracy of teamwork assessment in the operating room. Am Surg 2008;74(9):817-23.

92. Corrado J, Topley $\mathbf{K}$, Cracknell A. Improving the efficacy of elderly patients' hospital discharge through multi-professional safety briefings and behavioural change. BMJ Qual Improv Rep. 2015;4(1).

93. Gale RC, Asch SM, Taylor T, et al. The most used and most helpful facilitators for patient-centered medical home implementation. Implement Sci 2015;10:52.

94. Stansfield T, Parker R, Masson N, et al. The Endovascular Preprocedural Run Through and Brief: A Simple Intervention to Reduce Radiation Dose and Contrast Load in Endovascular Aneurysm Repair Vasc Endovasc Surg 2016;50(4):241-6.

95. Thanapongsathorn W, Jitsopa J, Wongviriyakorn O. Interprofessional preoperative briefing enhances surgical teamwork satisfaction and decrease operative time: a comparative study in abdominal operation. J Med Assoc Thail 2012;95 Suppl 12:S8-14.

96. Bennett SC, Finer N, Halamek LP, et al. Implementing Delivery Room Checklists and Communication Standards in a Multi-Neonatal ICU Quality Improvement Collaborative. Jt Comm J Qual Patient Saf 2016;42(8):369-76.

97. Kazana I, Pencak Murphy M. Implementing a patient-centered walking program for residents in long-term care: A quality improvement project. J Am Assoc Nurse Pract 2018;30(7):383-91.

98. Marshall DA, Manus DA. A team training program using human factors to enhance patient safety. AORN J 2007;86(6):994-1011.

99. Shaughnessy EE, White C, Shah SS, et al. Implementation of Postoperative Respiratory Care for Pediatric Orthopedic Patients. Pediatrics. 2015;136(2):e505-12.

100. Ansryan LZ, Aronow HU, Borenstein JE, et al. Systems Addressing Frail Elder Care: Description of a Successful Model. J Nurs Adm 2018;48(1):11-7.

101. Girdwood ST, Sellas M, Courter J, et al. Improving timely transition of parenteral to enteral antibiotics in paediatric patients with pneumonia and cellulitis using quality improvement methods. BMJ Open Qual 2017;6:A18-A9.

102. Gordon R, Ashley A, Banerjee T, et al. IHI ID 19 Improving family-care team communication during routine $\mathrm{CF}$ clinic visits. BMJ Open $\mathrm{Gual}$ 2018;7(Suppl 1):A24-A7.

103. Lingard L, Regehr G, Cartmill C, et al. Evaluation of a preoperative team briefing: a new communication routine results in improved clinical practice. BMJ Qual Saf 2011;20(6):475-82.

104. McBeth CL, Durbin-Johnson B, Siegel EO. Interprofessional Huddle: One Children's Hospital's Approach to Improving Patient Flow. Pediatr Nurs 2017;43(2):71-6. 
105. Mott A, Kafka S, Sutherland A. Assessing pharmaceutical care needs of paediatric in-patients: A team based approach. Arch Dis Child 2016;101(9):e2.

106. Parthiban A, Warta A, Marshall JA, et al. Improving Wait Time for Patients in a Pediatric Echocardiography Laboratory - a Quality Improvement Project. Pediatr Qual Saf 2018;3(3):e083.

107. Price T. Abstract TP405: Raising the bar, lowering the target. International Stroke Conference, 2016. ATP405.

108. Shivananda S, Gupta S, Thomas S, et al. Impact of a dedicated neonatal stabilization room and process changes on stabilization time. J Perinatol 2017;37(2):162-7.

109. Beardsley JR, Schomberg RH, Heatherly SJ, et al. Implementation of a Standardized Discharge Time-out Process to Reduce Prescribing Errors at Discharge. Hosp Pharm 2013;48(1):39-47.

110. Bell C, Jackson J, Shore H. P3 S.a.f.e. - the positive impact of 'druggles' on prescribing standards and patient safety within the neonatal intensive care environment. Arch Dis Child. 2018;103(2):e2.

111. Bhavee P, Rachel I, Pramodh V. P28 Reducing medication errors - a tripartite approach. small steps - better outcomes. Arch Dis Child. 2018;103(2):e1.

112. Keiffer S, Marcum G, Harrison S, et al. Reduction of medication errors in a pediatric cardiothoracic intensive care unit. $\mathrm{J}$ Nurs Care Qual 2015;30(3):212-9.

113. Phadnis J, Templeton-Ward O. Inadequate Preoperative Team Briefings Lead to More Intraoperative Adverse Events. J Patient Saf 2018;14(2):82-6.

114. AHC Media LLC. Team huddles improve LOS, core measures. Hosp Case Manag 2013;21(2):23-4.

115. Beck MJ, Okerblom D, Kumar A, et al. Lean intervention improves patient discharge times, improves emergency department throughput and reduces congestion. Hosp Pract 2016;44(5):252-9.

116. Chan A, Vadera S. 110 Neurosurgery Morning Huddle Reduces Costs and Increases Patient Satisfaction. Neurosurgery. 2017;64(CN_suppl_1):222-3.

117. Chan AY, Vadera S. Implementation of interdisciplinary neurosurgery morning huddle: cost-effectiveness and increased patient satisfaction. $\mathrm{J}$ Neurosurg 2018; 128(1):258-61

118. Patel H, Morduchowicz S, Mourad M. Using a Systematic Framework of Interventions to Improve Early Discharges. Jt Comm J Qual Patient Saf 2017;43(4):189-96.

119. Gams B, Neerland C, Kennedy S. Reducing Primary Cesareans: An Innovative Multipronged Approach to Supporting Physiologic Labor and Vaginal Birth. J Perinat Neonatal Nurs 2019;33(1):52-60.

120. Leone RM, Adams RJ. Safety Standards: Implementing Fall Prevention Interventions and Sustaining Lower Fall Rates by Promoting the Culture of Safety on an Inpatient Rehabilitation Unit. Rehabil Nurs 2016;41(1):26-32.

121. Neily J, Mills PD, Young-Xu Y, et al. Association between implementation of a medical team training program and surgical mortality. JAMA. 2010;304(15):1693-700.

122. Zubkoff L, Neily J, Quigley $\mathbf{P}$, et al. Preventing Falls and Fall-Related Injuries in State Veterans Homes: Virtual Breakthrough Series Collaborative. J Nurs Care Qual 2018;33(4):334-40.

123. Brass SD, Olney G, Glimp R, et al. Using the Patient Safety Huddle as a Tool for High Reliability. Jt Comm J Qual Patient Saf 2018;44(4):21926.

124. Storey J, Mustin L, Madsen N, et al. Improving the transfer of medically complex patients from the cardiac intensive care unit to the cardiology inpatient ward. BMJ Qual Saf 2015;24(11):737-8.

125. Reiter-Palmon R, Kennel V, Allen JA, et al. Naturalistic Decision Making in After-Action Review Meetings: The Implementation of and Learning from Post-Fall Huddles. J Occup Organ Psychol 2015;88(2):322-40

126. Thompson H, Vago T, Bell AM. Labor and Birth Improvements at Mercy Hospital St. Louis. J Obstet Gynecol Neonatal Nurs. 2015;44(s1):S14-S.

127. Wagner LM, Huijbregts M, Sokoloff LG, et al. Implementation of Mental Health Huddles on Dementia Care Units. Can J Aging 2014;33(3):235-45.

128. Cropper DP, Harb NH, Said PA, et al. Implementation of a patient safety program at a tertiary health system: A longitudinal analysis of interventions and serious safety events. J Healthc Risk Manag 2018;37(4):17-24.

129. Fridman V. The Effectiveness of Nurse-Driven Early Mobility Protocol. Seton Hall University DNP Final Projects. 2017;22.

130. Hansell K, Kirby EM. Team Engagement and Improvement Through Huddles. J Obstet Gynecol Neonatal Nurs 2015;44(s1):S46-S7.
131. Hebert $\mathbf{J}$. Improving hand hygiene through a multimodal approach. Nurs Manag 2015;46(11):27-30.

132. Simmons SF, Coelho CS, Sandler A, et al. A System for Managing Staff and Quality of Dementia Care in Assisted Living Facilities. J Am Geriatr Soc 2018;66(8):1632-7.

133. Sykes L, Sinha S, Hegarty J, et al. Reducing acute kidney injury incidence and progression in a large teaching hospital. BMJ Open Qual 2018;7(4):e000308.

134. Guo M, Tardif G, Bayley M. Medical Safety Huddles in Rehabilitation: A Novel Patient Safety Strategy. Arch Phys Med Rehabil 2018;99(6):12179.

135. McClead RE Jr, Catt C, Davis JT, et al. An internal quality improvement collaborative significantly reduces hospital-wide medication error related adverse drug events. J Pediatr. 2014;165(6):12229.e1.

136. Morvay S, Lewe D, Stewart B, et al. Medication event huddles: a tool for reducing adverse drug events. Jt Comm J Qual Patient Saf 2014;40(1):39-45

137. Fetzer M, Humphrey R, Stack-Simone S, et al. Pediatric catheter associated urinary tract infection reduction - an achievable goal. BMJ Gual Saf 2015;24(11):733-4.

138. Dewan $\mathbf{M}$, Wolfe $\mathbf{H}$, Lin $\mathbf{R}$, et al. Impact of a Safety Huddle-Based Intervention on Monitor Alarm Rates in Low-Acuity Pediatric Intensive Care Unit Patients. J Hosp Med 2017;12(8):652-7.

139. Zubkoff L, Neily J, Quigley P, et al. Virtual Breakthrough Series, Part 2: Improving Fall Prevention Practices in the Veterans Health Administration. Jt Comm J Qual Patient Saf. 2016;42(11):497-ap12.

140. Cummings B, Kelleher A, Mansoor Z, et al. 127: Reduce pediatric intensive care unit (PICU) rounds inefficiency through a preround huddle. Crit Care Med 2013;41(12):A25.

141. Monash B, Najafi N, Mourad M, et al. Standardized Attending Rounds to Improve the Patient Experience: A Pragmatic Cluster Randomized Controlled Trial. J Hosp Med 2017;12(3):143-9.

142. Nagelkerk J, Thompson ME, Bouthillier M, et al. Improving outcomes in adults with diabetes through an interprofessional collaborative practice program. $\mathrm{J}$ Interprofessional Care

143. Resar R, Nolan K, Kaczynski D, et al. Using real-time demand capacity management to improve hospitalwide patient flow. Jt Comm J Qual Patient Saf 2011;37(5):217-27.

144. Tielbur BR, Rice Cella DE, Currie A, et al. Discharge huddle outfitted with mobile technology improves efficiency of transitioning stroke patients into follow-up care. Am J Med Qual 2015;30(1):36-44.

145. Turner P. Implementation of TeamSTEPPS in the emergency department. Crit Care Nurs Q 2012;35(3):208-12.

146. Lisbon D, Allin D, Cleek C, et al. Improved Knowledge, Attitudes, and Behaviors After Implementation of TeamSTEPPS Training in an Academic Emergency Department: A Pilot Report. Am J Med Qual 2016;31(1):86-90

147. O'Connor T. Implementation of a pediatric behavioral staffing algorithm in an acute hospital: a best practice implementation project. JBI Database System Rev Implement Rep 2017;15(11):2799-814.

148. Taylor J, Barker A, Hill H, et al. Improving person-centered mobility care in nursing homes: a feasibility study. Geriatr Nurs 2015;36(2):98105.

149. Biernacki PJ, Champagne MT, Peng S, et al. Transformation of Care: Integrating the Registered Nurse Care Coordinator into the PatientCentered Medical Home. Popul Health Manag 2015;18(5):330-6.

150. Howard K, Huster J, Hodash G, et al. Improving fall rates using bedside debriefings and reflective emails: One unit's success story. Medsurg Nurs 2018;27(6):388-91

151. Shapiro J, Venkata A, Ochieng P, et al. 573: The Emergency Department to ICU Quality and Safety Project: Formal Handoff/ Huddle to Improve Care. Crit Care Med 2013;41(12):A140.

152. Shepherd EG, Kelly TJ, Vinsel JA, et al. Significant Reduction of Central-Line Associated Bloodstream Infections in a Network of Diverse Neonatal Nurseries. J Pediatr. 2015;167(1):41-6.e1-3.

153. Mullan PC, Macias CG, Hsu D, et al. A novel briefing checklist at shift handoff in an emergency department improves situational awareness and safety event identification. Pediatr Emerg Care 2015;31(4):231-8.

154. Pannick S, Archer S, Johnston MJ, et al. Translating concerns into action: a detailed qualitative evaluation of an interdisciplinary intervention on medical wards. BMJ Open 2017;7(4):e014401.

155. Sharma G, Wong D, Arnaoutakis DJ, et al. Systematic identification and management of barriers to vascular surgery patient discharge time of day. J Vasc Surg 2017;65(1):172-8. 
156. Blumenthal E, Carranza L, Sim MS. Improving Safety on Labor and Delivery Through Team Huddles and Teamwork Training [25H]. Obstet Gynecol 2016; 127:72S-3S.

157. Hastings SE, Suter E, Bloom J, et al. Introduction of a team-based care model in a general medical unit. BMC Health Serv Res 2016;16:245.

158. Dandoy CE, Hariharan S, Weiss B, et al. Sustained reductions in time to antibiotic delivery in febrile immunocompromised children: results of a quality improvement collaborative. BMJ Qual Saf 2016;25(2):100-9.

159. Pisegna L, Pyka J. An Interdisciplinary Approach to Improving Exclusive Breast Milk Feeding Rate at Discharge. J Obstet Gynecol Neonatal Nurs 2014;43:S61-S2.

160. Weintraub B, Jensen K, Colby $\mathbf{K}$. Improving hospitalwide patient flow at Northwest Community Hospital. Managing Patient Flow in Hospitals: Strategies and Solution, 2nd ed. Oak Brook: Joint Commission Resources. 2010:129-51.

161. AHC Media LLC. Two-stage screening tool improves identification of young sepsis patients in ED. ED Manag. 2017;29(12).

162. Pannick S, Athanasiou T, Long SJ, et al. Translating staff experience into organisational improvement: the HEADS-UP stepped wedge, cluster controlled, non-randomised trial. BMJ Open 2017;7(7):e014333.

163. van der Sluijs AF, van Slobbe-Bijlsma ER, Goossens A, et al. Reducing errors in the administration of medication with infusion pumps in the intensive care department: A lean approach. SAGE Open Med 2019;7:2050312118822629.

164. Wells T, Rockafellow S, Holler M, et al. Description of pharmacist-led quality improvement huddles in the patient-centered medical home model. J Am Pharm Assoc. 2018;58(6):667-72.e2.

165. Balamuth F, Alpern ER, Abbadessa MK, et al. Improving Recognition of Pediatric Severe Sepsis in the Emergency Department: Contributions of a Vital Sign-Based Electronic Alert and Bedside Clinician Identification. Ann Emerg Med. 2017;70(6):759-68.e2.

166. Cruz LC, Fine JS, Nori S. Barriers to discharge from inpatient rehabilitation: a teamwork approach. Int $\mathrm{J}$ Health Care Qual Assur 2017;30(2):137-47.

167. Hodges KT, Gilbert JH. Rising above risk: Eliminating infant falls. Nurs Manag 2015;46(12):28-32.

168. Hoke LM, Guarracino D. Beyond Socks, Signs, and Alarms: A Reflective Accountability Model for Fall Prevention. Am J Nurs 2016;116(1):42-7.

169. Menon S, Singh H, Giardina TD, et al. Safety huddles to proactively identify and address electronic health record safety. J Am Med Inform Assoc 2017;24(2):261-7.

170. Phillips J, Hebish LJ, Mann S, et al. Engaging Frontline Leaders and Staff in Real-Time Improvement. Jt Comm J Qual Patient Saf 2016;42(4):170-83.

171. Plouffe JA. Spacelabs Innovative Project Award winner-2007. Solar system of safety. Dynamics. 2010;21(3):20-1.

172. Ryckman FC, Yelton PA, Anneken AM, et al. Redesigning intensive care unit flow using variability management to improve access and safety. Jt Comm J Qual Patient Saf 2009;35(11):535-43.
173. Tetuan T, Ohm R, Kinzie L, et al. Does Systems Thinking Improve the Perception of Safety Culture and Patient Safety? J Nurs Regul 2017;8(2):31-9.

174. Nilsen P. Making sense of implementation theories, models and frameworks. Implement Sci 2015; 10:53.

175. Edbrooke-Childs J, Hayes J, Sharples E, et al. Development of the Huddle Observation Tool for structured case management discussions to improve situation awareness on inpatient clinical wards. BMJ Qual Saf 2018;27:365-72.

176. Ratcliffe TA, Crabtree MA, Palmer RF, et al. Service and Education The Association Between Workload, Patient Complexity, and Teaching on Internal Medicine Inpatient Services. J Gen Intern Med 2018;33:44954

177. Gonzalo JD, Chuang $\mathbf{C H}$, Huang G, et al. The return of bedside rounds: an educational intervention. J Gen Intern Med 2010;25:792-8.

178. Levine D, Gadivemula J, Kutaimy R, et al. Analysis of patient safety messages delivered and received during clinical rounds. BMJ Open Qual 2020;9.

179. Norrie C, Moriarty J, Lipman V, et al. A qualitative study of handovers at shift changeovers in five care homes for older people in England. Int $\mathrm{J}$ Older People Nursing. 2020:e12339.

180. Bakon S, Wirihana L, Christensen M, et al. Nursing handovers: An integrative review of the different models and processes available. Int $\mathrm{J}$ Nurs Pract 2017;23.

181. Kitson AL, Muntlin Athlin A, Elliott J, et al. What's my line? A narrative review and synthesis of the literature on Registered Nurses' communication behaviours between shifts. J Adv Nurs 2014;70:122842 .

182. Ogrinc G, Davies L, Goodman D, et al. SQUIRE 2.0 (Standards for QUality Improvement Reporting Excellence): revised publication guidelines from a detailed consensus process. BMJ Qual Saf. 2016;25:98692.

183. Davies P, Walker AE, Grimshaw JM. A systematic review of the use of theory in the design of guideline dissemination and implementation strategies and interpretation of the results of rigorous evaluations. Implement Sci 2010;5:14.

184. Kaplan HC, Brady PW, Dritz MC, et al. The influence of context on quality improvement success in health care: a systematic review of the literature. Milbank Q 2010;88:500-59.

185. Knudsen SV, Laursen HVB, Johnsen SP, et al. Can quality improvement improve the quality of care? A systematic review of reported effects and methodological rigor in plan-do-study-act projects. BMC Health Serv Res. 2019;19:683.

186. Tabak RG, Khoong EC, Chambers DA, et al. Bridging research and practice: models for dissemination and implementation research. Am J Prev Med 2012;43:337-50.

Publisher's Note Springer Nature remains neutral with regard to jurisdictional claims in published maps and institutional affiliations. 